\title{
Mobile Interaction with Web Services through Associated Real World Objects
}

\author{
${ }^{1}$ Gregor Broll, ${ }^{2}$ John Hamard, ${ }^{2}$ Massimo Paolucci, ${ }^{1}$ Markus Haarländer, ${ }^{2}$ Matthias \\ Wagner, ${ }^{1}$ Sven Siorpaes, ${ }^{3}$ Enrico Rukzio, ${ }^{4}$ Albrecht Schmidt, ${ }^{2}$ Kevin Wiesner \\ ${ }^{1}$ Media Informatics Group, University of Munich, Germany \\ ${ }^{2}$ DoCoMo Euro-Labs, Germany \\ ${ }^{3}$ Computing Department, Lancaster University, UK \\ ${ }^{4}$ Fraunhofer IAIS, Sankt Augustin and B-IT, University of Bonn, Germany \\ gregor.broll@ifi.Imu.de, \{markus, sven\}@hcilab.org, rukzio@comp.lancs.ac.uk, \\ \{paolucci, hamard, wagner, wiesner\}@docomolab-euro.com, \\ albrecht.schmidt@iais.fraunhofer.de
}

\begin{abstract}
The proposed demonstration is based on the work performed in the PERCI project and presents a generic framework to access and interact with Web Services through mobile interaction with real world objects. The demonstration will put a focus on the front-end of the framework that comprises augmented posters for mobile ticketing as well as a mobile client application for the interaction with associated services. By supporting Physical Mobile Interaction techniques such as Touching or Pointing, the framework tries to make mobile service interaction more intuitive and shift its focus from the menus of mobile devices to augmented physical objects.
\end{abstract}

\section{Keywords}

Physical Mobile Interaction, Semantic Web Services, visual marker recognition, NFC, Touching, Pointing, Direct Input

\section{INTRODUCTION: MOBILE SERVICES AND PHYSICAL MOBILE INTERACTION}

Despite the advancements in Mobile Computing, the usage of Web Services in the mobile domain is still not as advanced, widespread and established as in Desktop Computing. Mobile devices almost naturally suffer from different constraints like small displays, fiddly keyboards and glutted menus which complicate mobile interaction with Web Services and limit the presentation of their contents.

In this context, Physical Mobile Interaction [1] can help facilitate mobile interaction with Web Services: This interaction paradigm benefits from the dissemination of technologies such as RFID [2], NFC (Near Field Communication) [3], Bluetooth or visual

Permission to make digital or hard copies of all or part of this work for personal or classroom use is granted without fee provided that copies are not made or distributed for profit or commercial advantage and that copies bear this notice and the full citation on the first page. To copy otherwise, or republish, to post on servers or to redistribute to lists, requires prior specific permission and/or a fee.

Mobile HCI'07, September 9-12, 2007, Singapore.

Copyright 2007 ACM 978-1-59593-862-6.......\$5.00 markers [4] that allow the augmentation of everyday objects like posters or leaflets with additional information for their association with e.g. web pages or services. Complementary, mobile phones increasingly have available the technologies to extract this information from physical objects, process it and use it in different applications. Physical Mobile Interaction eases the constrained interaction with mobile phones and partially replaces it with the more intuitive interaction with physical objects. Instead of browsing nested and glutted menus of mobile applications, users can simply touch or point at the things they want to interact with. Similarly, they can discover services, connect and interact with them by touching NFC-tags or taking pictures of visual markers.

In this context, the proposed demonstration will present a generic framework that takes advantage of Physical Mobile Interaction in order to leverage and facilitate mobile interaction with Web Services. The framework was developed in the PERCI project (PERvasive ServiCe Interaction) [5], a collaboration between the University of Munich and NTT DoCoMo Euro-Labs. The demonstration will put a focus on the front-end of this framework that comprises augmented posters for mobile ticketing and a mobile client application for the interaction with associated services. The next section will provide an overview of the proposed contribution while section 3 will specify details for the setup of the demonstration.

\section{SUMMARY OF CONTRIBUTION}

The PERCI-framework leverages mobile interaction with Web Services as it automatically generates adaptable mobile interfaces from their semantically extended descriptions. In a first step, the framework generates an abstract interface description which is then transformed into different interfaces on specific mobile platforms. Currently, the framework supports the generation of HTML and Java ME interfaces. These mobile interfaces again support Physical Mobile Interaction with objects from the real world that provide information for the interaction with associated Web Services.

The interfaces on mobile clients are complemented by physical objects that are augmented with information for service discovery and invocation. Mobile devices act as mediators between services 
and physical objects, as they extract information from them and use it for the interaction with their associated services. As a prerequisite, Physical Mobile Interaction pushes features and options for service interaction off mobile phone menus and maps them to information on physical objects. As a result, the focus of interaction with the mobile device is shifted and extended to the interaction with the real world.

This kind of Physical Mobile Interaction with services does not rely on hard-coded procedures but uses a generic typing mechanism that matches information captured through Physical Mobile Interaction with requested service input parameters. Every item of information that conforms to the typing scheme of the PERCI framework can be used with its services and either identify them or serve as a parameter for their invocation. Using a common information typing scheme enables different interaction techniques besides simple service invocation. For example, crossobject interaction makes it possible to invoke a service discovered on one physical object with parameter information acquired from another physical object.

The demonstration will present different front-ends for using the framework and invoking its services. It will comprise a mobile client application with HTML- and Java ME-interfaces for Physical Mobile Interaction with 2 posters for mobile ticketing. The first poster implements a simplified way to buy tickets for a public transportation system (see Figure 1). Instead of having to understand the complicated ticketing system, inexperienced users only have to select options for the stations they want to start their journey from, their destination, the number of passengers as well as the duration of the journey in order to have appropriate tickets suggested.

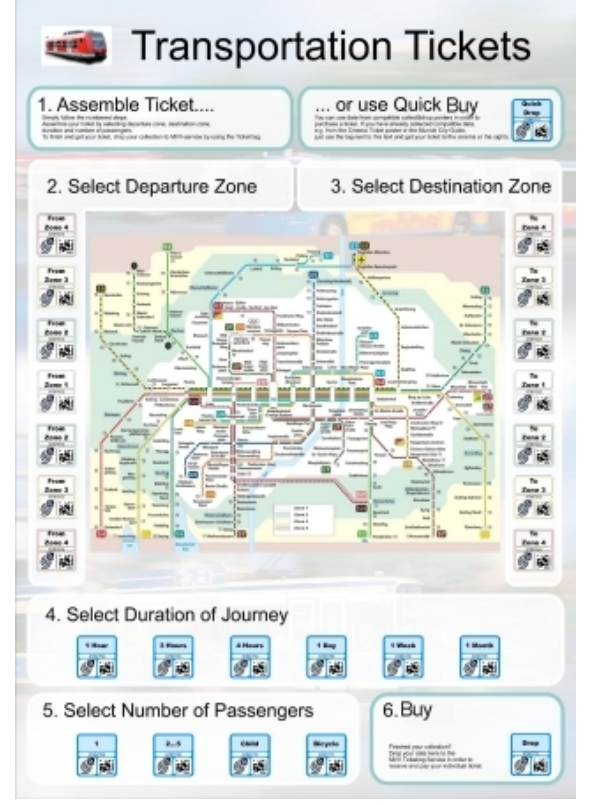

Figure 1: Augmented poster for transportation ticketing

The second poster allows users to purchase movie tickets and includes appropriate options (movie title, cinema name, number of tickets and preferred timeslots) together with a selection of values (see Figure 2).

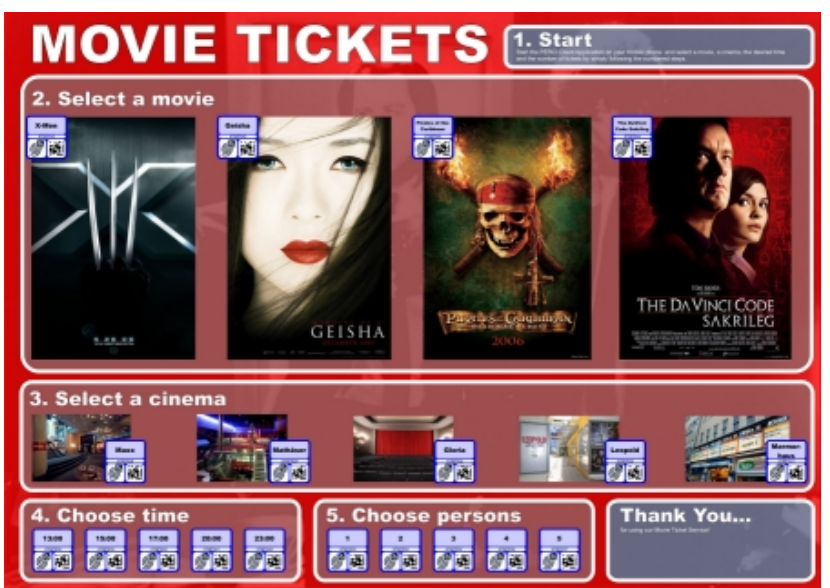

Figure 2: Augmented poster for movie ticketing

Each option on the posters is augmented with a numeric identifier, a NFC tag and a visual marker. They contain or reference the information that is represented by the option, e.g. the name of a movie, the number of tickets or different stations. The mobile client application supports the Physical Mobile Interaction techniques Touching (using NFC, see Figure 3a), Pointing (using the recognition of visual markers, see Figure 3b) and Direct Input of numeric identifiers (see Figure 3c) for which a user simply types a numeric identifier into a form. Users interact with the posters by selecting different options with their mobile devices in an arbitrary order using the supported interaction techniques. In the process, they extract information from the posters and use it for the invocation of associated services.

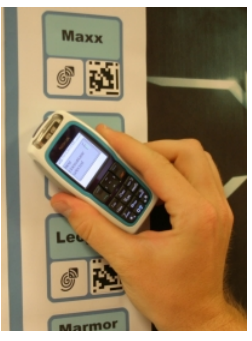

a)

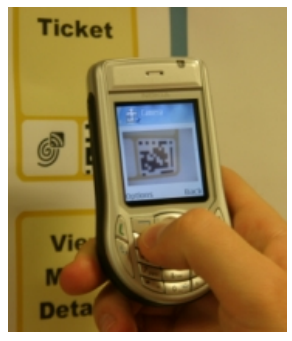

b)

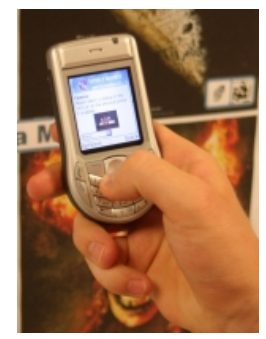

c)
Figure 3. Physical Mobile Interaction techniques Touching (a), Pointing (b) and Direct Input (c)

User tests highlighted the usefulness and usability of this prototype. Participants particularly described it as an intuitive, cheap and enjoyable solution, which could for instance replace existing automats with paper posters. Please see [6] for details.

\section{DESCRIPTION AND SETUP OF DEMONSTRATION}

The setup of the proposed demonstration will consist of 2 prototype posters for mobile ticketing that have been prepared for the Physical Mobile Interaction techniques Touching (using NFC), Pointing (using visual markers) and the direct input of numeric identifiers into a form. The augmented posters as well as suitable mobile phones (e.g. Nokia 6131 NFC) will be provided in order to show how the mobile client application interacts with the framework and its services. For the setup of the demo, a vertical 
space of approximately 2 by 2 meters will be required for putting up the posters.

Visitors will be able to invoke ticketing services from the posters through mobile phones using both a simple HTML-browser and a Java ME client application that supports the interaction techniques Touching and Pointing. They will see how interfaces for the service invocation - though based on the same abstract interface description - can be rendered differently depending on which mobile device and interaction technique they use.

It has to be noted that the PERCI framework has also been demonstrated at PERCOM 07 and WIMA 07/ NFC Competition. Since the framework is constantly evolved, the demonstration for MobileHCI will comprise the latest versions of the mobile client application and the posters. It will also showcase new features such as Physical Mobile Interaction with services across different objects.

\section{REFERENCES}

[1] Gregor Broll, Sven Siorpaes, Enrico Rukzio, Massimo Paolucci, John Hamard, Matthias Wagner, Albrecht Schmidt.
Supporting Mobile Service Usage through Physical Mobile Interaction. PerCom 2007, White Plains, NY, USA, March 2007.

[2] Roy Want. An Introduction to RFID Technology. IEEE Pervasive Computing, vol. 5, pp. 25-33, 2006.

[3] NFC Forum. http://www.nfc-forum.org/.

[4] Michael Rohs, Beat Gfeller. Using Camera-Equipped Mobile Phones for Interacting with Real-World Objects. In: Alois Ferscha, Horst Hoertner, Gabriele Kotsis (Eds.): Advances in Pervasive Computing, Austrian Computer Society (OCG), Vienna, Austria, 2004.

[5] PERCI (PERvasive ServiCe Interaction) website. http://www.hcilab.org/projects/perci/

[6] Gregor Broll, Sven Siorpaes, Enrico Rukzio, Massimo Paolucci, John Hamard, Matthias Wagner, Albrecht Schmidt. Comparing Techniques for Mobile Interaction with Objects from the Real World. Workshop Permid 2007 in conjunction with Pervasive 2007, Toronto, Ontario, Canada, May 13 2007. 